\title{
Evaluation of organochlorine pesticide residues in human serum from an urban and two rural populations in Portugal
}

\author{
Susana Cruz, Celeste Lino*, Maria Irene Silveira \\ Group of Bromatology, Center of Pharmaceutical Studies, Faculty of Pharmacy, University of Coimbra, \\ Rua do Norte 3000-295 Coimbra, Portugal
}

Received 7 November 2002; accepted 1 May 2003

\begin{abstract}
Organochlorine pesticide residues were measured in human serum from an urban and two rural populations in Portugal, in an attempt to evaluate the contamination level of Portuguese population. Serum levels of 12 residues were determined using a validated methodology that included gas chromatography-electron-capture detection. It was made an attempt to point out the differences of contamination between rural and urban populations; and among these, if it could be established a relation with sex and with age of individuals. $p, p^{\prime} \mathrm{DDE}, \alpha$-hexachlorocyclohexane $(\mathrm{HCH})$, $p, p^{\prime} \mathrm{DDD}$, and $\beta-\mathrm{HCH}$ were the most frequently identified residues. $p, p^{\prime} \mathrm{DDE}$ concentrations ranged from undetected to $390.5 \mu \mathrm{g} / 1$ in urban samples, and from undetected to $43.5 \mu \mathrm{g} / 1$ and to $171.2 \mu \mathrm{g} / 1$ in both rural samples. Maximum $\alpha-\mathrm{HCH}$ concentration level was $114.4 \mu \mathrm{g} / \mathrm{l}$ in urban samples, 261.3 and $45.5 \mu \mathrm{g} / 1$ in both rural samples. Mean total DDT levels were always higher than mean total $\mathrm{HCH}$ levels. About $p, p^{\prime} \mathrm{DDE}$, in all three populations, the majority of the results above the limit of quantification were found among female sex. The analysis of different aged groups showed that younger groups continue to reveal contamination. Comparing obtained results with others from Europe, Asia and America, it was observed that Portugal is between the highest levels of contamination.
\end{abstract}

(c) 2003 Elsevier B.V. All rights reserved.

Keywords: Organochlorine; Hexachlorocyclohexane; DDT; Rural; Urban

\section{Introduction}

Organochlorine pesticides are a class of chemicals that came into widespread use in the late 1940s. Despite being banned in industrialized countries since the 1970 s, or subjected to restrictions in use in many others, they persist to this day in the environment. In Portugal most of

*Corresponding author. Tel.: +51-239859994; fax: +51239827126.

E-mail address: cmlino@ci.uc.pt (C. Lino). organochlorine pesticide residues have been prohibited in 1988 (Decree 660/88) and this year it was legislated the prohibition on lindane's use in agriculture. With the exception of occupationally exposed individuals, most exposure to these chemicals occurs via dietary intake (DeVoto et al., 1998; Ahlborg et al., 1995), especially food of animal origin, but also through water, ambient and indoor air, dust and soil (Covaci et al., 2002; Dua et al., 2001; Manirakiza et al., 2002). These lipophilic compounds accumulate and even bio- 
magnify their concentration along the food chain, especially in fatty food (Manirakiza et al., 2002). Some published reports (Ahlborg et al., 1995; DeVoto et al., 1998; Hanaoka et al., 2002; Manirakiza et al., 2002) suggest that serum levels of organochlorines are related to the consumption of various foods. The current background body burden of organochlorine pesticides is still of concern, all over the world, because of their sex-hormonelike properties, more specifically estrogenic properties that point out these chemicals as endocrine disrupters and as suspected carcinogens (Ahlborg et al., 1995; Amaral Mendes, 2002; Charlier and Plomteux, 2002; DeVoto et al., 1998; Frías et al., 2001; Gammon et al., 2002; Sonnenschein et al., 1995). Recent reports suggested an association between exposure to pesticides and different types of human cancer (Amaral Mendes, 2002; Safi, 2002). The facts that such residues persist in adipose tissue over a period of many decades, are endocrine disrupters, cause immune suppression, and inhibit various enzymes, lead to higher susceptibility to cancer onset (Iscan et al., 2002; Wolff et al., 2000; Safi, 2002; Saxena et al., 1981).

Biological monitoring of exposure can be carried out by determination of intact compounds or their metabolites in blood, serum, plasma and urine (Aprea et al., 2002). Thus, human serum is one of the biological materials that can be conveniently obtained for the study of body burden following chronic exposure (Waliszewski et al., 1999a).

Our aim was to determine the body burden of 12 organochlorine pesticide residues in individuals from an urban population, and from two rural populations which are placed in an area of intense agricultural activity, and for that, with higher environmental exposure, by measuring their concentrations in blood serum. We intended to provide useful data on contamination levels in a region of Europe that has not been well studied.

This study directs attention to possible differences between, rural and urban residence, gender and aged groups, and higher serum organochlorine levels. All the samples were analysed for the following residues: hexachlorocyclohexane $(\mathrm{HCH})$ isomers $(\alpha, \beta, \gamma)$, aldrin and dieldrin, heptachlor epoxide (HE), hexachlorobenzene (HCB), 1,1,1trichloro-2,2-bis ( $p$-chorophenyl) ethane ( $p, p^{\prime}$ -
DDT), 1,1,1-trichloro-2-(o-chlorophenyl)-2-( $p$ chlorophenyl) ethane (o, $p^{\prime}$-DDT), 1,1-dichloro2,2-bis ( $p$-chlorophenyl) ethylene ( $p, p^{\prime}$-DDE) and 1,1-dichloro-2,2-bis ( $p$-chlorophenyl) ethane ( $p, p^{\prime}$ DDD), and endosulfan sulphate.

\section{Experimental}

\subsection{Sampling and description of populations}

The present study was carried out between the year of 2001 and 2002 in the central region of Portugal, in the area of Coimbra, the most important city of this region, placed between Lisbon and Oporto. The river Mondego crosses Coimbra. Samples are proceeding from 203 presumably healthy volunteers, chosen by a random procedure, 44 urban residents, living in Coimbra, and 159 rural residents, 70 from the farming village of Verride and 89 from the farming village of Ereira. These two villages are situated on the Mondego valley, far from Coimbra, approximately $30 \mathrm{~km}$. Verride is on the left bank at a higher level as opposed of Ereira which stays on the right bank at river level.

The Coimbra population comprised 27 women and 17 men, aged between 20 and 60; the Verride population comprised 42 women and 28 men, aged between 20 and 94; and finally, the Ereira population comprised 64 women and 25 men, aged between 19 and 90 .

All volunteers were asked to complete a rapid questionnaire about age, body mass index $(\mathrm{kg} /$ $\mathrm{m}^{2}$ ), parity, clinical history, occupation and contact with pesticides. All of them were considered nonoccupationally exposed.

The blood samples, approximately $10 \mathrm{ml}$, were collected by venipuncture and the serum was separated by centrifugation and frozen at $-20{ }^{\circ} \mathrm{C}$ in glass vials (prewashed with $n$-hexane).

\subsection{Chemical analysis}

The analysis of 12 organochlorine pesticide residues was performed according to the method 2 of Lino et al. (1998) modified by the addition of $0.5 \mathrm{ml}$ of methanol to $1 \mathrm{ml}$ of serum before the extraction. Briefly, the extraction of organochlorine residues was made with $2 \times 5 \mathrm{ml}$ of $n$-hexane- 
acetone $(90+10)$, shaken for $1 \mathrm{~min}$ on a Vortex mixer and centrifuged at $1250 \times g$ for $5 \mathrm{~min}$. The extract was transferred to a Florisil SPE cartridge previously added with $1 \mathrm{~cm}$ of sodium sulphate. Two different eluents were used: $\mathrm{E}_{1} 6 \mathrm{ml} n$-hexane and $\mathrm{E}_{2} 6 \mathrm{ml} n$-hexane- $\mathrm{Cl}_{2} \mathrm{CH}_{2}(5+1)$. The eluates were concentrated to $1 \mathrm{ml}$ and then, quantification and confirmation of results were made using gas chromatography-electron-capture detection $\left({ }^{63} \mathrm{Ni}\right)$ with two columns, DB-5 and DB-17.

\section{Results}

This study revealed that mean concentration level of all analysed compounds, excepting $\mathrm{HCH}$ isomers, was higher in urban samples (Table 1). The median concentrations of all the compounds were below the quantification limit. We considered total $\mathrm{HCH}\left(\sum-\mathrm{HCH}\right)$ as the sum of the three isomers $(\alpha, \beta, \gamma)$ and total DDT ( $\sum$-DDT) as the sum of the four compounds: $p, p^{\prime} \mathrm{DDE}, p, p^{\prime} \mathrm{DDD}$, $p, p^{\prime} \mathrm{DDT}, o, p^{\prime} \mathrm{DDT}$. Because the distribution of serum organochlorine levels was not normal, differences between two groups or more were tested by Student's $t$-test or the analysis of variance. A $P$-value less than 0.05 was considered to be statistically significant.

In case of $\mathrm{HCH}$ isomers, it was observed that mean total $\mathrm{HCH}$ levels were higher in the Verride population, $13 \pm 36.6 \mu \mathrm{g} / 1$ and 16 samples were above the limit of quantification (LOQ). For mean total $\mathrm{HCH}$ levels in Coimbra and Verride, the isomer $\alpha$ was the one that contributed maximum, while in Ereira was the isomer $\beta$.

Mean concentration levels of DDT isomers and analogues were higher in samples proceeding from Coimbra, however, the highest number of results above the LOQ has been found among Ereira samples. $p, p^{\prime} \mathrm{DDE}$ levels ranged from non-detectable to $390.5 \mu \mathrm{g} / \mathrm{l}$, in Coimbra, with the majority of results being below the LOQ. The highest value found for $o, p^{\prime}$ DDT residue belongs to an individual from the Ereira population with $256.7 \mu \mathrm{g} / \mathrm{l}$. $p, p^{\prime}$ DDT was detected above the LOQ only in 2 samples from the urban population with the maximum value of $814.9 \mu \mathrm{g} / \mathrm{l}$. Among DDT isomers and analogues $p, p^{\prime} \mathrm{DDE}$ was the residue with more results above the LOQ.
Comparing mean levels of total $\mathrm{HCH}$ with total DDT, it was observed that, in Coimbra and in Ereira, total DDT levels were nine times higher than the first ones, $93.5 \pm 140.9 \mu \mathrm{g} / 1$ vs. $10 \pm 22.8$ $\mu \mathrm{g} / \mathrm{l}$, in Coimbra, and $56 \pm 50 \mu \mathrm{g} / 1$ vs. $6.1 \pm 8.6$ $\mu \mathrm{g} / \mathrm{l}$, in Ereira. In Verride these values were three times higher, $43.9 \pm 9.7 \mu \mathrm{g} / \mathrm{l}$, for total DDT, vs. $13 \pm 36.6 \mu \mathrm{g} / \mathrm{l}$, for total $\mathrm{HCH}$.

The concentrations of aldrin, dieldrin and HE were always below the LOQ in rural samples. In urban samples these residues have been detected above the LOQ in a small number of samples. HCB was detected above the LOQ in 4 samples from Coimbra, with the maximum value of 393.3 $\mu \mathrm{g} / \mathrm{l}$, and in 1 sample from Ereira with $28.2 \mu \mathrm{g} /$ 1. Endosulfan sulphate was detected above the LOQ in 1 sample from Coimbra, with $547.6 \mu \mathrm{g} /$ 1, and in another one from Ereira with $108.8 \mu \mathrm{g}$ / 1.

The most frequently detected residue was $p, p^{\prime} \mathrm{DDE}$, which was found in concentrations above the LOQ in 27 samples from Ereira, in 13 from Verride and in 9 from Coimbra. In the three analysed populations, $o, p^{\prime} \mathrm{DDT}$ was more often detected than $p, p^{\prime}$ DDT, which appeared in concentrations above the LOQ only in two samples from Coimbra. Comparing number of results above the LOQ of total $\mathrm{HCH}$ with total DDT, it was observed a prevalence of DDT in Coimbra and Ereira. However, in Verride, total $\mathrm{HCH}$ had 16 results above the LOQ and total DDT had 14.

In fact, the statistical analysis with an ANOVA, trying to correlate higher levels of each compound with place of origin, either urban or rural, showed significant differences $(P<0.05)$ for $p, p^{\prime} \mathrm{DDE}$, total DDT, HCB, aldrin, dieldrin and HE. These results are expressed in Table 7.

The analysis of samples concerning to individuals sex is expressed in Table 2. First analysis of this table shows that the majority of the results above the LOQ were found in males from the urban population and in females from both rural populations. In respect to mean $\mathrm{HCH}$ concentration levels, higher levels were found in males, 17.4 $\mu \mathrm{g} / \mathrm{l}$ for total $\mathrm{HCH}$, than in females, $5.2 \mu \mathrm{g} / \mathrm{l}$, from the urban population. In the rural population of Verride, mean concentration of isomer $\alpha$ and of total $\mathrm{HCH}$ were also higher in males than in 
Table 1

concentration $(x)(\mu \mathrm{g} / \mathrm{l})$, standard deviation (S.D.), ranges and number of samples above the LOQ of organochlorine pesticides detected in human serum samples from an urban population, Coimbra and two rural populations, in Verride and Ereira, Portugal

\begin{tabular}{|c|c|c|c|c|c|c|c|c|c|}
\hline & \multicolumn{3}{|c|}{ Coimbra $(N=44)$} & \multicolumn{3}{|c|}{ Verride $(N=70)$} & \multicolumn{3}{|c|}{ Ereira $(N=89)$} \\
\hline & $X \pm$ S.D. & Min-máx & $>$ LOQ & $x \pm$ S.D. & Min-máx & $>$ LOQ & $x \pm$ S.D. & Min-máx & $>$ LOQ \\
\hline \multicolumn{10}{|l|}{ Pesticide } \\
\hline$\alpha-\mathrm{HCH}$ & $7.6 \pm 22.8$ & $<3.2-114.4$ & 7 & $10.2 \pm 36.3$ & $<3.2-261.3$ & 11 & $2.2 \pm 4.7$ & $<3.2-45.5$ & 2 \\
\hline$\beta-\mathrm{HCH}$ & $1.6 \pm 0.3$ & $<3.2-3.5$ & 1 & $2.2 \pm 1.9$ & $<3.2-13.3$ & 8 & $3.3 \pm 6.9$ & $<3.2-44.3$ & 7 \\
\hline$\gamma-\mathrm{HCH}$ & $0.68 \pm 0.70$ & $<1.08-4.8$ & 2 & $0.6 \pm 0.4$ & $<1.08-3.5$ & 2 & $0.7 \pm 1.0$ & $<1.08-7.5$ & 2 \\
\hline$\sum-\mathrm{HCH}$ & $10 \pm 22.8$ & $<1.08^{\mathrm{a}}-114.4$ & 9 & $13 \pm 36.6$ & $<1.08^{\mathrm{a}}-265.8$ & 16 & $6.1 \pm 8.6$ & $<1.08^{\mathrm{a}}-45.5$ & 9 \\
\hline$p, p^{\prime} \mathrm{DDE}$ & $28.6 \pm 75$ & $<12.5-390.5$ & 9 & $9.5 \pm 8.1$ & $<12.5-43.5$ & 13 & $14.6 \pm 20.6$ & $<12.5-171.2$ & 27 \\
\hline$p, p^{\prime} \mathrm{DDD}$ & $12 \pm 17.4$ & $<15-95.3$ & 3 & $7.95 \pm 2.7$ & $<15-25.9$ & 2 & $11.9 \pm 23.2$ & $<15-199.1$ & 8 \\
\hline$o, p^{\prime} \mathrm{DDT}$ & $15.4 \pm 26.6$ & $<15-141.0$ & 4 & $7.7 \pm 1.6$ & $<15-20.7$ & 1 & $10.8 \pm 26.5$ & $<15-256.7$ & 4 \\
\hline$p, p^{\prime} \mathrm{DDT}$ & $37.5 \pm 120$ & $<37.5-814.9$ & 2 & $18.8 \pm 0.0$ & $<37.5-<37.5$ & 0 & $18.8 \pm 0.0$ & $<37.5-<37.5$ & 0 \\
\hline$\sum$-DDT & $93.5 \pm 140.9$ & $<12.5^{\mathrm{a}}-814.9$ & 15 & $43.9 \pm 9.7$ & $<12.5^{\mathrm{a}-70.7}$ & 14 & $56 \pm 50$ & $<12.5^{\mathrm{a}}-427.9$ & 33 \\
\hline $\mathrm{HCB}$ & $20 \pm 64.3$ & $<12.5-393.3$ & 4 & $6.3 \pm 0.0$ & $<12.5-<12.5$ & 0 & $6.5 \pm 2.3$ & $<12.5-28.2$ & 1 \\
\hline Aldrin & $17.4 \pm 65.3$ & $<5-372.9$ & 3 & $2.5 \pm 0.0$ & $<5-<5$ & 0 & $2.5 \pm 0.0$ & $<5-<5$ & 0 \\
\hline Dieldrin & $22.3 \pm 61.9$ & $<14.5-356.4$ & 3 & $7.3 \pm 0.0$ & $<14.5-<14.5$ & 0 & $7.3 \pm 0.0$ & $<14.5-<14.5$ & 0 \\
\hline H.E. & $14.8 \pm 36.6$ & $<12.5-239.1$ & 5 & $6.3 \pm 0.0$ & $<12.5-<12.5$ & 0 & $6.3 \pm 0.0$ & $<12.5-<12.5$ & 0 \\
\hline Endosulfan sulphate & $19.8 \pm 81.4$ & $<15-547.6$ & 1 & $7.5 \pm 0.0$ & $<15-<15$ & 0 & $8.6 \pm 10.7$ & $<15-108.8$ & 1 \\
\hline
\end{tabular}

If the concentration was below the LOQ it was set to $50 \%$ of that limit when the mean and S.D. was calculated.

${ }^{\text {a }}$ We have considered the lowest LOQ of the added compounds. 
Table 2

concentration $(x)(\mu \mathrm{g} / 1)$, S.D. and number of samples above the LOQ of organochlorine pesticides detected in human serum samples of both sexes from an urban population, Coimbra, and two rural populations, in Verride and Ereira, Portugal

\begin{tabular}{|c|c|c|c|c|c|c|c|c|c|c|c|c|}
\hline & \multicolumn{4}{|l|}{ Coimbra } & \multicolumn{4}{|l|}{ Verride } & \multicolumn{4}{|l|}{ Ereira } \\
\hline & \multicolumn{2}{|c|}{ Females $(N=27)$} & \multicolumn{2}{|c|}{ Males $(N=17)$} & \multicolumn{2}{|c|}{ Females $(N=42)$} & \multicolumn{2}{|c|}{ Males $(N=28)$} & \multicolumn{2}{|c|}{ Females $(N=64)$} & \multicolumn{2}{|c|}{ Males $(N=25)$} \\
\hline & $x \pm$ S.D. & $>$ LOQ & $x \pm$ S.D. & $>$ LOQ & $x \pm$ S.D. & $>$ LOQ & $x \pm$ S.D. & $>$ LOQ & $x \pm$ S.D. & $>$ LOQ & $x \pm$ S.D. & $>$ LOQ \\
\hline \multicolumn{13}{|l|}{ Pesticide } \\
\hline$\alpha-\mathrm{HCH}$ & $3.1 \pm 7.5$ & 2 & $14.8 \pm 35.0$ & 5 & $6.7 \pm 20.0$ & 7 & $15.5 \pm 52.1$ & 4 & $2.3 \pm 5.5$ & 1 & $1.8 \pm 1.1$ & 1 \\
\hline$\beta-\mathrm{HCH}$ & $1.6 \pm 0.0$ & 0 & $1.7 \pm 0.5$ & 1 & $2.3 \pm 2.2$ & 5 & $2.0 \pm 1.3$ & 3 & $3.5 \pm 7.8$ & 5 & $2.7 \pm 4.0$ & 2 \\
\hline$\gamma-\mathrm{HCH}$ & $0.5 \pm 0.0$ & 0 & $0.9 \pm 1.1$ & 2 & $0.6 \pm 0.5$ & 2 & $0.5 \pm 0.0$ & 0 & $0.6 \pm 0.8$ & 1 & $0.8 \pm 1.4$ & 1 \\
\hline$\sum-\mathrm{HCH}$ & $5.2 \pm 7.5$ & 2 & $17.4 \pm 34.8$ & 7 & $9.7 \pm 20.3$ & 10 & $18.0 \pm 52.5$ & 6 & $6.5 \pm 9.7$ & 6 & $5.3 \pm 4.8$ & 3 \\
\hline$p, p^{\prime} \mathrm{DDE}$ & $27.8 \pm 63.0$ & 7 & $29.8 \pm 93.0$ & 2 & $10.0 \pm 8.6$ & 9 & $8.7 \pm 7.4$ & 4 & $17.1 \pm 23.6$ & 23 & $8.3 \pm 5.5$ & 4 \\
\hline$p, p^{\prime} \mathrm{DDD}$ & $10.8 \pm 16.9$ & 1 & $13.9 \pm 18.4$ & 2 & $7.8 \pm 2.0$ & 1 & $8.2 \pm 3.5$ & 1 & $8.1 \pm 2.8$ & 3 & $21.5 \pm 42.6$ & 5 \\
\hline$o, p^{\prime} \mathrm{DDT}$ & $12.7 \pm 19.1$ & 2 & $19.6 \pm 35.8$ & 2 & $7.5 \pm 0.0$ & 0 & $8.0 \pm 2.5$ & 1 & $11.9 \pm 31.2$ & 3 & $8.1 \pm 3.0$ & 1 \\
\hline$p, p^{\prime} \mathrm{DDT}$ & $18.8 \pm 0.0$ & 0 & $67.4 \pm 192.8$ & 2 & $18.8 \pm 0.0$ & 0 & $18.8 \pm 0.0$ & 0 & $18.8 \pm 0.0$ & 0 & $18.8 \pm 0.0$ & 0 \\
\hline$\sum-\mathrm{DDT}$ & $70.1 \pm 72.6$ & 8 & $130.7 \pm 205.9$ & 7 & $44.0 \pm 8.7$ & 10 & $43.6 \pm 11.2$ & 4 & $55.7 \pm 52.6$ & 24 & $56.7 \pm 43.6$ & 9 \\
\hline $\mathrm{HCB}$ & $28.4 \pm 81.6$ & 3 & $6.8 \pm 2.1$ & 1 & $6.3 \pm 0.0$ & 0 & $6.3 \pm 0.0$ & 0 & $6.6 \pm 2.7$ & 1 & $6.3 \pm 0.0$ & 0 \\
\hline Aldrin & $24.7 \pm 82.4$ & 2 & $5.9 \pm 13.8$ & 1 & $2.5 \pm 0.0$ & 0 & $2.5 \pm 0.0$ & 0 & $2.5 \pm 0.0$ & 0 & $2.5 \pm 0.0$ & 0 \\
\hline Dieldrin & $27.7 \pm 76.5$ & 2 & $13.6 \pm 26.0$ & 1 & $7.3 \pm 0.0$ & 0 & $7.3 \pm 0.0$ & 0 & $7.3 \pm 0.0$ & 0 & $7.3 \pm 0.0$ & 0 \\
\hline H.E. & $16.6 \pm 44.9$ & 3 & $11.8 \pm 17.5$ & 2 & $6.3 \pm 0.0$ & 0 & $6.3 \pm 0.0$ & 0 & $6.3 \pm 0.0$ & 0 & $6.3 \pm 0.0$ & 0 \\
\hline
\end{tabular}

If the concentration was below the LOQ it was set to $50 \%$ of that limit when the mean and S.D. was calculated. 
Table 3

Mean concentration $(x)(\mu \mathrm{g} / 1)$, S.D. and number of samples above the LOQ of organochlorine pesticides detected in human serum samples of different aged groups from an urban population, in Coimbra, Portugal

\begin{tabular}{|c|c|c|c|c|c|c|}
\hline & \multicolumn{6}{|c|}{ Coimbra $(N=44)$} \\
\hline & \multicolumn{2}{|c|}{$20-39$ aged group $(N=24)$} & \multicolumn{2}{|c|}{$40-49$ aged group $(N=9)$} & \multicolumn{2}{|c|}{$50-59$ aged group $(N=11)$} \\
\hline & $x \pm$ S.D. & $>$ LOQ & $x \pm$ S.D. & $>$ LOQ & $x \pm$ S.D. & $>$ LOQ \\
\hline \multicolumn{7}{|l|}{ Pesticide } \\
\hline$\alpha-\mathrm{HCH}$ & $12.6 \pm 30.3$ & 6 & $1.6 \pm 0.0$ & 0 & $1.8 \pm 0.5$ & 1 \\
\hline$\beta-\mathrm{HCH}$ & $1.6 \pm 0.0$ & 0 & $1.8 \pm 0.7$ & 1 & $1.6 \pm 0.0$ & 0 \\
\hline$\gamma-\mathrm{HCH}$ & $0.7 \pm 0.9$ & 1 & $0.5 \pm 0.0$ & 0 & $0.7 \pm 0.6$ & 1 \\
\hline$\sum-\mathrm{HCH}$ & $14.9 \pm 30.3$ & 7 & $4.0 \pm 0.7$ & 1 & $4.1 \pm 1.1$ & 1 \\
\hline$p, p^{\prime} \mathrm{DDE}$ & $32.0 \pm 81.0$ & 6 & $6.3 \pm 0.0$ & 0 & $39.5 \pm 92.2$ & 3 \\
\hline$p, p^{\prime} \mathrm{DDD}$ & $11.2 \pm 17.9$ & 1 & $19.6 \pm 24.5$ & 2 & $7.5 \pm 0.0$ & 0 \\
\hline op'DDT & $18.9 \pm 32.9$ & 3 & $15.5 \pm 23.9$ & 1 & $7.5 \pm 0.0$ & 0 \\
\hline$p, p^{\prime} \mathrm{DDT}$ & $18.8 \pm 0.0$ & 0 & $18.8 \pm 0.0$ & 0 & $93.9 \pm 239.3$ & 2 \\
\hline$\sum-\mathrm{DDT}$ & $80.8 \pm 90.8$ & 8 & $60.1 \pm 42.2$ & 2 & $148.4 \pm 245.5$ & 5 \\
\hline HCB & $23.6 \pm 78.9$ & 3 & $27.3 \pm 63.2$ & 1 & $6.3 \pm 0.0$ & 0 \\
\hline Aldrin & $12.1 \pm 46.8$ & 1 & $8.8 \pm 19.0$ & 1 & $36.2 \pm 111.7$ & 1 \\
\hline Dieldrin & $26.3 \pm 73.6$ & 2 & $7.3 \pm 0.0$ & 0 & $25.8 \pm 61.4$ & 1 \\
\hline $\mathrm{HE}$ & $9.8 \pm 14.4$ & 2 & $9.0 \pm 8.2$ & 1 & $30.3 \pm 69.9$ & 2 \\
\hline Endosulfan Sulphate & $30.0 \pm 110.2$ & 1 & $7.5 \pm 0.0$ & 0 & $7.5 \pm 0.0$ & 0 \\
\hline
\end{tabular}

If the concentration was below the LOQ it was set to $50 \%$ of that limit when the mean and S.D. was calculated.

females, $18.0 \mu \mathrm{g} / 1$ for total $\mathrm{HCH}$ in males vs. 9.7 $\mu \mathrm{g} / 1$ in females. The opposite situation was observed in the rural population of Ereira, mean total $\mathrm{HCH}$ concentration in females was higher, $6.5 \mu \mathrm{g} / 1$, than in males, $5.3 \mu \mathrm{g} / 1$.

About $p, p^{\prime} \mathrm{DDE}$, in all three populations, the majority of the results above the LOQ were found among female sex. In respect to mean $p, p^{\prime} \mathrm{DDE}$ concentration levels of rural samples, higher values were observed in females rather than in males, $10.0 \mu \mathrm{g} / 1$ vs. $8.7 \mu \mathrm{g} / 1$ in Verride, and $17.1 \mu \mathrm{g} / 1$ vs. $8.3 \mu \mathrm{g} / 1$ in Ereira.

In the three populations, mean concentration values of $p, p^{\prime} \mathrm{DDD}$ were higher in males. $o, p^{\prime} \mathrm{DDT}$ was detected with higher mean concentration in males from the Coimbra and Verride populations, and in females from the Ereira population. $p, p^{\prime}$ DDT was detected only in 2 male samples from Coimbra.

Total DDT higher levels of average were detected in males, rather than in females, from the Coimbra population, 130.7 vs. $70.1 \mu \mathrm{g} / \mathrm{l}$. Both rural populations showed very similar values of mean total DDT levels for both sexes. The majority of the results of total DDT above the LOQ were found among females of the three populations.

The other residues, HCB, aldrin, dieldrin, HE and endosulfan sulphate mean concentration levels were always higher in females from the Coimbra population. In the other two populations their results were in most cases below the LOQ.

In order to compare each residue with sex of individuals the Student's $t$-test was used and did not indicate any significant difference for any of the 12 compounds in analysis.

Distributing the three populations in different aged groups (Tables 3-5) we were able to compare the same aged groups (20-39, 40-49, 50-59). Looking closely to Tables $3-5$ it was observed that in Coimbra, $\mathrm{HCH}$ isomers and its sum had higher mean levels in younger groups; in Ereira it happened the same, excepting for $\alpha-\mathrm{HCH}$, which was detected above the LOQ in samples from older groups. About $p, p^{\prime} \mathrm{DDE}$, in Coimbra, the highest frequency was obtained in the youngest group, although the highest average was obtained in the oldest one, $39.5 \mu \mathrm{g} / \mathrm{l}$; in Verride and Ereira both values were found in the oldest groups. In 
Table 4

Mean concentration $(x)(\mu \mathrm{g} / 1)$, and S.D. and number of samples above the LOQ of organochlorine pesticides detected in human serum samples of different aged groups from a rural population, in Verride, Portugal

\begin{tabular}{|c|c|c|c|c|c|c|c|c|c|c|c|c|c|c|}
\hline & \multicolumn{14}{|l|}{ Verride } \\
\hline & \multicolumn{2}{|c|}{$\begin{array}{l}20-39 \text { aged group } \\
(N=6)\end{array}$} & \multicolumn{2}{|c|}{$\begin{array}{l}40-49 \text { aged group } \\
(N=6)\end{array}$} & \multicolumn{2}{|c|}{$\begin{array}{l}50-59 \text { aged group } \\
(N=11)\end{array}$} & \multicolumn{2}{|c|}{$\begin{array}{l}60-69 \text { aged group } \\
(N=14)\end{array}$} & \multicolumn{2}{|c|}{$\begin{array}{l}70-79 \text { aged group } \\
(N=19)\end{array}$} & \multicolumn{2}{|c|}{$\begin{array}{l}80-89 \text { aged group } \\
(N=10)\end{array}$} & \multicolumn{2}{|c|}{$\begin{array}{l}» 90 \text { aged group } \\
(N=4)\end{array}$} \\
\hline & $x \pm$ S.D. & $>$ LOQ & $x \pm$ S.D. & $>$ LOQ & $x \pm$ S.D. & $>$ LOQ & $x \pm$ S.D. & $>$ LOQ & $x \pm$ S.D. & $>$ LOQ & $x \pm$ S.D. & $>$ LOQ & $x \pm$ S.D. & $>$ LOQ \\
\hline \multicolumn{15}{|l|}{ Pesticide } \\
\hline$\alpha-\mathrm{HCH}$ & $18.9 \pm 42.4$ & 1 & $3.5 \pm 4.6$ & 1 & $12.3 \pm 35.4$ & 1 & $20.7 \pm 69.3$ & 2 & $4.1 \pm 5.3$ & 5 & $7.4 \pm 18.2$ & 1 & $1.6 \pm 0.0$ & 0 \\
\hline$\beta-\mathrm{HCH}$ & $2 \pm 0.9$ & 1 & $1.6 \pm 0.0$ & 0 & $1.8 \pm 0.6$ & 1 & $2.2 \pm 1.6$ & 2 & $2.9 \pm 3.2$ & 3 & $1.6 \pm 0.0$ & 0 & $2.7 \pm 2.2$ & 1 \\
\hline$\gamma-\mathrm{HCH}$ & $0.5 \pm 0.0$ & 0 & $0.5 \pm 0.0$ & 0 & $0.7 \pm 0.4$ & 1 & $0.5 \pm 0.0$ & 0 & $0.7 \pm 0.7$ & 1 & $0.5 \pm 0.0$ & 0 & $0.5 \pm 0.0$ & 0 \\
\hline$\sum-\mathrm{HCH}$ & $21.4 \pm 42.2$ & 2 & $5.6 \pm 4.6$ & 1 & $14.7 \pm 35.7$ & 2 & $23.4 \pm 70.0$ & 3 & $7.6 \pm 7.2$ & 6 & $9.5 \pm 18.2$ & 1 & $4.9 \pm 2.2$ & 1 \\
\hline$p, p^{\prime} \mathrm{DDE}$ & $6.3 \pm 0.0$ & 0 & $6.3 \pm 0.0$ & 0 & $9.6 \pm 11.2$ & 1 & $11.5 \pm 10.7$ & 4 & $9.5 \pm 6.1$ & 5 & $8.7 \pm 5.2$ & 2 & $13.3 \pm 14.2$ & 1 \\
\hline$p, p^{\prime} \mathrm{DDD}$ & $7.5 \pm 0.0$ & 0 & $7.5 \pm 0.0$ & 0 & $7.5 \pm 0.0$ & 0 & $7.5 \pm 0.0$ & 0 & $8.5 \pm 4.2$ & 1 & $7.5 \pm 0.0$ & 0 & $10.8 \pm 6.6$ & 1 \\
\hline$o, p^{\prime} \mathrm{DDT}$ & $7.5 \pm 0$ & 0 & $7.5 \pm 0.0$ & 0 & $7.5 \pm 0.0$ & 0 & $7.5 \pm 0.0$ & 0 & $8.2 \pm 3.0$ & 1 & $7.5 \pm 0.0$ & 0 & $7.5 \pm 0.0$ & 0 \\
\hline$p, p^{\prime} \mathrm{DDT}$ & $18.8 \pm 0.0$ & 0 & $18.8 \pm 0.0$ & 0 & $18.8 \pm 0.0$ & 0 & $18.8 \pm 0.0$ & 0 & $18.8 \pm 0.0$ & 0 & $18.8 \pm 0.0$ & 0 & $18.8 \pm 0.0$ & 0 \\
\hline$\sum$-DDT & $40 \pm 0.0$ & 0 & $40 \pm 0.0$ & 0 & $43.4 \pm 11.2$ & 1 & $45.3 \pm 10.7$ & 4 & $44.9 \pm 11.9$ & 5 & $42.5 \pm 5.2$ & 2 & $50.4 \pm 13.5$ & 2 \\
\hline $\mathrm{HCB}$ & $6.3 \pm 0.0$ & 0 & $6.3 \pm 0.0$ & 0 & $6.3 \pm 0.0$ & 0 & $6.3 \pm 0.0$ & 0 & $6.3 \pm 0.0$ & 0 & $6.3 \pm 0.0$ & 0 & $6.3 \pm 0.0$ & 0 \\
\hline Aldrin & $2.5 \pm 0.0$ & 0 & $2.5 \pm 0.0$ & 0 & $2.5 \pm 0.0$ & 0 & $2.5 \pm 0.0$ & 0 & $2.5 \pm 0.0$ & 0 & $2.5 \pm 0.0$ & 0 & $2.5 \pm 0.0$ & 0 \\
\hline Dieldrin & $7.3 \pm 0.0$ & 0 & $7.3 \pm 0.0$ & 0 & $7.3 \pm 0.0$ & 0 & $7.3 \pm 0.0$ & 0 & $7.3 \pm 0.0$ & 0 & $7.3 \pm 0.0$ & 0 & $7.3 \pm 0.0$ & 0 \\
\hline H.E. & $6.3 \pm 0.0$ & 0 & $6.3 \pm 0.0$ & 0 & $6.3 \pm 0.0$ & 0 & $6.3 \pm 0.0$ & 0 & $6.3 \pm 0.0$ & 0 & $6.3 \pm 0.0$ & 0 & $6.3 \pm 0.0$ & 0 \\
\hline Endosulfan sulphate & $7.5 \pm 0.0$ & 0 & $7.5 \pm 0.0$ & 0 & $7.5 \pm 0.0$ & 0 & $7.5 \pm 0.0$ & 0 & $7.5 \pm 0.0$ & 0 & $7.5 \pm 0.0$ & 0 & $7.5 \pm 0.0$ & 0 \\
\hline
\end{tabular}

If the concentration was below the LOQ it was set to $50 \%$ of that limit when the mean and S.D. was calculated. 
Table 5

Mean concentration $(x)(\mu \mathrm{g} / 1)$ and S.D. and number of samples above the LOQ of organochlorine pesticides detected in human serum samples of different aged groups from a rural population, in Ereira, Portugal

\begin{tabular}{|c|c|c|c|c|c|c|c|c|c|c|c|c|}
\hline & \multicolumn{12}{|l|}{ Ereira } \\
\hline & \multicolumn{2}{|c|}{$\begin{array}{l}20-39 \text { aged group } \\
(N=12)\end{array}$} & \multicolumn{2}{|c|}{$\begin{array}{l}40-49 \text { aged group } \\
(N=4)\end{array}$} & \multicolumn{2}{|c|}{$\begin{array}{l}50-59 \text { aged group } \\
(N=13)\end{array}$} & \multicolumn{2}{|c|}{$\begin{array}{l}60-69 \text { aged group } \\
(N=28)\end{array}$} & \multicolumn{2}{|c|}{$\begin{array}{l}70-79 \text { aged group } \\
(N=20)\end{array}$} & \multicolumn{2}{|c|}{$\begin{array}{l}80-90 \text { aged group } \\
(N=12)\end{array}$} \\
\hline & $x \pm$ S.D. & $>$ LOQ & $x \pm$ S.D. & $>\mathrm{LOQ}$ & $x \pm$ S.D. & $>$ LOQ & $x \pm$ S.D. & $>$ LOQ & $x \pm$ S.D. & $>\mathrm{LOQ}$ & $x \pm$ S.D. & $>$ LOQ \\
\hline \multicolumn{13}{|l|}{ Pesticide } \\
\hline$\alpha-\mathrm{HCH}$ & $1.6 \pm 0.0$ & 0 & $1.6 \pm 0.0$ & 0 & $1.6 \pm 0.0$ & 0 & $3.2 \pm 8.3$ & 1 & $1.6 \pm 0.0$ & 0 & $2.1 \pm 1.6$ & 1 \\
\hline$\beta-\mathrm{HCH}$ & $5.2 \pm 10.0$ & 2 & $2.8 \pm 2.3$ & 1 & $4.9 \pm 11.8$ & 1 & $2.2 \pm 3.4$ & 1 & $3.7 \pm 6.9$ & 2 & $1.6 \pm 0.0$ & 0 \\
\hline$\gamma-\mathrm{HCH}$ & $1.7 \pm 2.6$ & 2 & $0.5 \pm 0.0$ & 0 & $0.5 \pm 0.0$ & 0 & $0.5 \pm 0.0$ & 0 & $0.5 \pm 0.0$ & 0 & $0.5 \pm 0.0$ & 0 \\
\hline$\sum-\mathrm{HCH}$ & $8.5 \pm 12.3$ & 2 & $4.9 \pm 2.3$ & 1 & $7.0 \pm 11.8$ & 1 & $6.0 \pm 8.8$ & 2 & $5.8 \pm 6.9$ & 2 & $4.2 \pm 1.6$ & 1 \\
\hline$p, p^{\prime} \mathrm{DDE}$ & $7.3 \pm 3.7$ & 1 & $6.3 \pm 0.0$ & 0 & $16.7 \pm 16.6$ & 5 & $11.6 \pm 10.9$ & 7 & $23.9 \pm 37.4$ & 9 & $14.0 \pm 9.8$ & 5 \\
\hline$p, p^{\prime} \mathrm{DDD}$ & $16 \pm 29.3$ & 1 & $7.5 \pm 0.0$ & 0 & $8.4 \pm 3.2$ & 1 & $14.7 \pm 36.2$ & 2 & $10.7 \pm 8.4$ & 3 & $8.3 \pm 2.8$ & 1 \\
\hline$o, p^{\prime} \mathrm{DDT}$ & $8.8 \pm 4.3$ & 1 & $7.5 \pm 0.0$ & 0 & $7.5 \pm 0.0$ & 0 & $8.2 \pm 3.9$ & 1 & $20.4 \pm 55.7$ & 2 & $7.5 \pm 0.0$ & 0 \\
\hline$p, p^{\prime} \mathrm{DDT}$ & $18.8 \pm 0.0$ & 0 & $18.8 \pm 0.0$ & 0 & $18.8 \pm 0.0$ & 0 & $18.8 \pm 0.0$ & 0 & $18.8 \pm 0.0$ & 0 & $18.8 \pm 0.0$ & 0 \\
\hline$\sum-\mathrm{DDT}$ & $50.8 \pm 33.5$ & 2 & $40 \pm 0.0$ & 0 & $51.3 \pm 18.4$ & 5 & $53.2 \pm 36.9$ & 9 & $73.8 \pm 91$ & 11 & $48.6 \pm 9.5$ & 6 \\
\hline $\mathrm{HCB}$ & $6.3 \pm 0.0$ & 0 & $6.3 \pm 0.0$ & 0 & $6.3 \pm 0.0$ & 0 & $6.3 \pm 0.0$ & 0 & $7.4 \pm 4.9$ & 1 & $6.3 \pm 0.0$ & 0 \\
\hline Aldrin & $2.5 \pm 0.0$ & 0 & $2.5 \pm 0.0$ & 0 & $2.5 \pm 0.0$ & 0 & $2.5 \pm 0.0$ & 0 & $2.5 \pm 0.0$ & 0 & $2.5 \pm 0.0$ & 0 \\
\hline Dieldrin & $7.3 \pm 0.0$ & 0 & $7.3 \pm 0.0$ & 0 & $7.3 \pm 0.0$ & 0 & $7.3 \pm 0.0$ & 0 & $7.3 \pm 0.0$ & 0 & $7.3 \pm 0.0$ & 0 \\
\hline H.E. & $6.3 \pm 0.0$ & 0 & $6.3 \pm 0.0$ & 0 & $6.3 \pm 0.0$ & 0 & $6.3 \pm 0.0$ & 0 & $6.3 \pm 0.0$ & 0 & $6.3 \pm 0.0$ & 0 \\
\hline Endosulfan Sulphate & $15.9 \pm 29.2$ & 1 & $7.5 \pm 0.0$ & 0 & $7.5 \pm 0.0$ & 0 & $7.5 \pm 0.0$ & 0 & $7.5 \pm 0.0$ & 0 & $7.5 \pm 0.0$ & 0 \\
\hline
\end{tabular}

If the concentration was below the LOQ it was set to $50 \%$ of that limit when the mean and S.D. was calculated. 
Table 6

Comparison of organochlorine pesticide residue mean levels $(\mu \mathrm{g} / \mathrm{l})$ in blood serum samples of different countries

\begin{tabular}{|c|c|c|c|c|c|c|c|c|c|c|c|c|c|c|c|c|c|c|}
\hline \multirow[t]{2}{*}{ Pesticide } & \multirow{2}{*}{$\begin{array}{l}\text { Pakistan } \\
\text { Krawinkel } \\
\text { et al. } \\
\text { (1989) }\end{array}$} & \multirow{2}{*}{$\begin{array}{l}\text { Spain } \\
\text { Gómez- } \\
\text { Catalán } \\
\text { et al. } \\
(1987) \\
\text { Guardino } \\
\text { et al. } \\
(1996)^{a}\end{array}$} & \multirow{2}{*}{$\begin{array}{l}\text { Italy } \\
\text { Leoni } \\
\text { et al. } \\
(1989)\end{array}$} & \multirow{2}{*}{$\begin{array}{l}\text { Sweden }^{\mathrm{c}} \\
\text { Wicklund } \\
\text { Glynn et } \\
\text { al. } \\
(2000)\end{array}$} & \multirow[t]{2}{*}{$\begin{array}{l}\text { Canada }^{\mathrm{a}, \mathrm{d}, \mathrm{c}} \\
\text { Mes } \\
(1992)\end{array}$} & \multicolumn{2}{|c|}{$\begin{array}{l}\text { Germany } \\
\text { DeVoto et al. } \\
\text { (1998) }\end{array}$} & \multirow{2}{*}{$\begin{array}{l}\text { Mexico } \\
\text { Waliszewski } \\
\text { et al. } \\
(1999 \text { a) }\end{array}$} & \multirow[t]{2}{*}{$\begin{array}{l}\text { USA } \\
\text { Stellman } \\
\text { et al. } \\
\text { (1998) }\end{array}$} & \multicolumn{3}{|c|}{$\begin{array}{l}\text { India }^{\mathrm{f}} \\
\text { Dua et al. (2001) }\end{array}$} & \multicolumn{2}{|c|}{$\begin{array}{l}\text { Belgium } \\
\text { Charlier and } \\
\text { Plomteux (2002) }\end{array}$} & \multirow{2}{*}{$\begin{array}{l}\text { Japan } \\
\text { Hanaoka } \\
\text { et al. } \\
(2002)\end{array}$} & \multicolumn{3}{|c|}{$\begin{array}{l}\text { Portugal } \\
\text { This study } 2002\end{array}$} \\
\hline & & & & & & Men & Women & & & March & July & Nov. & Men & Women & & Coimbra & Verride & Ereira \\
\hline$\alpha-\mathrm{HCH}$ & 0.08 & & & 1.2 & 0.04 & 0.9 & 0.5 & 0.1 & & 0.59 & 0.54 & 0.18 & & & & 7.6 & 10.2 & 2.2 \\
\hline$\beta-\mathrm{HCH}$ & 1.39 & & & 48.6 & 0.13 & 2.94 & 2.9 & 1.4 & 0.82 & 1.51 & 1.89 & 0.94 & & & 0.54 & 1.6 & 2.2 & 3.3 \\
\hline$\gamma-\mathrm{HCH}$ & 0.29 & & & 1.8 & & 2.52 & 1.9 & 0.2 & & 0.04 & 0.69 & 0.2 & & & & 0.7 & 0.6 & 0.7 \\
\hline$\sum-\mathrm{HCH}$ & & & & & & & & 1.6 & & 2.14 & 3.12 & 1.32 & & & & 9.95 & 13.0 & 6.1 \\
\hline$p, p^{\prime} \mathrm{DDE}$ & 8.58 & $3.3^{\mathrm{a}}$ & 4.57 & 808.9 & 0.87 & & & 14.5 & 4.72 & 1.33 & 1.55 & 1.37 & & & & 28.6 & 9.5 & 14.6 \\
\hline$p, p^{\prime} \mathrm{DDD}$ & & $0.5^{\mathrm{a}}$ & & 2.8 & & & & 0.3 & & 0.37 & 0.91 & 0.64 & & & & 11.98 & 7.95 & 11.9 \\
\hline$o, p^{\prime} \mathrm{DDT}$ & & & & 2.2 & 0.13 & & & 1.0 & 0.22 & nd & 0.01 & nd & & & & 15.4 & 7.7 & 10.8 \\
\hline$p, p^{\prime} \mathrm{DDT}$ & 0.61 & $0.3^{\mathrm{a}}$ & & 20.2 & 0.03 & & & 1.8 & 0.22 & 1.03 & 4.46 & 2.58 & & & & 37.5 & 18.8 & 18.8 \\
\hline$\sum$-DDT & & $4.1^{\mathrm{a}}$ & 5.59 & & & 14.8 & 15.4 & 16.4 & & 2.73 & 6.92 & 4.59 & 8.2 & 6.1 & 6.3 & 93.5 & 43.9 & 56.0 \\
\hline $\mathrm{HCB}$ & & 11.1 & 1.49 & 83.1 & & 10.2 & 11.9 & 1.0 & 0.21 & & & & 3.6 & 3.7 & 0.24 & 20.0 & 6.3 & 6.5 \\
\hline
\end{tabular}


Table 7

Application of variance analysis (Turkey), with the factor place (Coimbra, Verride and Ereira) and the dependent variables, the organochlorine pesticide residues

\begin{tabular}{|c|c|c|c|c|}
\hline & (I) Place & (J) Place & $\begin{array}{l}\text { Mean difference } \\
(\mathrm{I}-\mathrm{J})\end{array}$ & $P$-value \\
\hline$p, p^{\prime} \mathrm{DDE}$ & 1 & $\begin{array}{l}2 \\
3 \\
1 \\
3 \\
1 \\
2\end{array}$ & $\begin{array}{c}19.1364^{*} \\
14.0046 \\
-19.1364^{*} \\
-5.1318 \\
-14.0046 \\
5.1318\end{array}$ & $\begin{array}{l}0.024 \\
0.110 \\
0.024 \\
0.670 \\
0.110 \\
0.670\end{array}$ \\
\hline$\sum$-DDT & 2 & $\begin{array}{l}2 \\
3 \\
1 \\
3 \\
1 \\
2\end{array}$ & $\begin{aligned} & 49.635^{*} \\
& 37.493^{*} \\
- & 49.635^{*} \\
- & 12.142 \\
- & 37.493^{*} \\
& 12.142\end{aligned}$ & $\begin{array}{l}0.002 \\
0.017 \\
0.002 \\
0.556 \\
0.017 \\
0.556\end{array}$ \\
\hline $\mathrm{HCB}$ & 1 & $\begin{array}{l}2 \\
3 \\
1 \\
3 \\
1 \\
2\end{array}$ & $\begin{array}{c}13.7700^{*} \\
13.5234^{*} \\
-13.7700^{*} \\
-0.2466 \\
-13.5234 * \\
0.2466\end{array}$ & $\begin{array}{l}0.046 \\
0.039 \\
0.046 \\
0.999 \\
0.039 \\
0.999\end{array}$ \\
\hline Aldrin & 1 & $\begin{array}{l}2 \\
3 \\
1 \\
3 \\
1 \\
2\end{array}$ & $\begin{array}{c}14.925^{*} \\
14.925^{*} \\
-14.925^{*} \\
0.000 \\
-14.925^{*} \\
0.000\end{array}$ & $\begin{array}{l}0.030 \\
0.022 \\
0.030 \\
1.000 \\
0.022 \\
1.000\end{array}$ \\
\hline Dieldrin & 1 & $\begin{array}{l}2 \\
3 \\
1 \\
3 \\
1 \\
2\end{array}$ & $\begin{array}{c}15.0011^{*} \\
15.0011^{*} \\
-15.0011^{*} \\
0.0000 \\
-15.0011^{*} \\
0.0000\end{array}$ & $\begin{array}{l}0.020 \\
0.014 \\
0.020 \\
1.000 \\
0.014 \\
1.000\end{array}$ \\
\hline HE & 1 & $\begin{array}{l}2 \\
3 \\
1 \\
3 \\
1 \\
2\end{array}$ & $\begin{array}{c}8.5066^{*} \\
8.5066^{*} \\
-8.5066^{*} \\
0.0000 \\
-8.5066^{*} \\
0.0000\end{array}$ & $\begin{array}{l}0.027 \\
0.020 \\
0.027 \\
1.000 \\
0.020 \\
1.000\end{array}$ \\
\hline
\end{tabular}

SPSS software was used for statistical analysis: *statistically significant $(P<0.05, N=203)$; place 1 -Coimbra population; place 2 -Verride population; place 3 -Ereira population.

the Coimbra population, $p, p^{\prime} \mathrm{DDD}$ was found in younger groups; in Ereira, it was detected with higher mean concentration level in the youngest group, $16 \mu \mathrm{g} / \mathrm{l}$, but with more results above the LOQ in older groups; in Verride the highest average was seen in the oldest group, $10.8 \mu \mathrm{g} / 1$. About $o, p^{\prime}$ DDT it was observed that higher levels were detected in younger groups from the urban population. The two results above the LOQ for $p, p^{\prime} \mathrm{DDT}$ were found in the oldest group of Coimbra population.

Total DDT higher mean concentration values were most of the times observed in older groups. The higher number of samples with concentrations above the LOQ was also found among older groups in both rural populations, while in urban samples this number was higher in the youngest group.

In the Coimbra population, $\mathrm{HCB}$, dieldrin and endosulfan sulphate highest levels were found in the younger group. Aldrin and HE were detected with higher mean concentration level in the oldest group from this population.

The analysis of variance taking into account the factor age did not reveal any statistically significant differences between any of the aged groups in analysis and organochlorine residues levels.

\section{Discussion}

The present analysis for the Portuguese population was consistent with numerous studies in other populations that have shown the difficulty in analysing human data due to the diversity of parameters to take into account, and also have shown that organochlorine pollutants' problematic is far from being totally understood (Ahlborg et al., 1995; Gammon et al., 2002; Koppen et al., 2002; Manirakiza et al., 2002).

The results have shown that $\gamma-\mathrm{HCH}$ (lindane) was the less detected of the three $\mathrm{HCH}$ isomers. Mean total $\mathrm{HCH}$ concentration was higher in the Verride population, having the maximum contribution of the isomer $\alpha$. These are interesting findings once isomers $\alpha$ and $\beta$ are essentially noninsecticidal, they probably appear as a result of application of lindane impure formulations either in agriculture, for crop protection, either in shampoos and lotions for treatment of scabies and lice. This is of concern due to high persistence of these two isomers, especially $\beta-\mathrm{HCH}$, in the environment, and in human body (Ahlborg et al., 1995; Lino and Silveira, 1996). 
Our study has shown higher serum levels of total DDT in urban samples; this correlation reached a high statistically significant level $(P \leqslant 0.002)$. This situation can be due to higher consumption of animal origin foods and dairy products by this population (Ahlborg et al., 1995; DeVoto et al., 1998). Just like it happens in many other countries from the Occidental Europe, Portugal imports animal and vegetable origin foods from Asia and Latin America where DDT is still in use (González, 1998; Shaw, 1998). Urban populations preferentially consume these imported products, once they have higher accessibility to this kind of products while rural populations tend to consume the products they grow.

It is important to note that $p, p^{\prime} \mathrm{DDT}$ is classified by the IARC as group 2B, 'possibly carcinogenic to humans' (IARC Monographs, 1991), and that it was found in $1 \%$ of total analysed samples with the maximum value of $814.9 \mu \mathrm{g} / 1$.

The results of HCB showed that it was highly detected in urban samples $(P \leqslant 0.046)$, probably as a result of dietary habits, such as meat, fish and poultry (Ahlborg et al., 1995), as well as, beef and lamb (DeVoto et al., 1998). Although HCB is no longer used as a fungicide, it is still produced as a by-product and contaminant in other chlorinated chemicals, it is released in the environment by the discharge of plastic (Ando et al., 1985; Gómez-Catalán et al., 1987) and it has also been found in fly ash from fires or incinerators (DeVoto et al., 1998); therefore HCB continues to enter in the food chain. HCB also occurs in the human body as a metabolite of $\mathrm{HCH}$ (Ahlborg et al., 1995). This organochlorine compound is highly lipophilic and very persistent and also a potent inductor of porphyria (Ando et al., 1985). In animals, HCB is carcinogenic and also is metabolised to pentachlorophenol, which is a very toxic substance (Gómez-Catalán et al., 1987).

Aldrin and dieldrin were detected only in a few number of urban samples $(P \leqslant 0.03)$. However, it is not clear why aldrin continues to appear in human serum, once it is rapidly metabolised to dieldrin, in the environment and in human body (Ahlborg et al., 1995). The fact that they have been detected could be explained by a recent exposure (Frías et al., 2001).
HE is the most persistent metabolite of heptachlor, which was used against soil insects, termites and mosquitoes. We have only detected it in the urban population $(P \leqslant 0.027)$. Once more this is probably a result of exposure by the ingestion of contaminated food, such as meat, fish and dairy products (Ahlborg et al., 1995; DeVoto et al., 1998).

We observed a prevalence among men rather than women of higher levels of total $\mathrm{HCH}$. Other studies, in Germany, Japan and Sene-Gambian region, also reported higher levels of $\mathrm{HCH}$ isomers for males (DeVoto et al., 1998; Hanaoka et al., 2002; Manirakiza et al., 2002).

Just like in other studies from the United States, Belgium and Egypt (Ahlborg et al., 1995; Charlier and Plomteux, 2002; Soliman et al., 1997), we have also observed mean total DDT levels in men higher than those of women. These results could suggest that lactation is a route of excretion, as there are studies that indicate the transfer of organochlorine residues to breastmilk (Harris et al., 2001; Waliszewski et al., 1999b), or suggest that there are other gender differences in storage and metabolism (Ahlborg et al., 1995).

Mean HCB concentration was higher in females than in males; other studies also have found higher mean HCB concentration levels in females (Charlier and Plomteux, 2002; DeVoto et al., 1998).

Despite all this, and because we have not found any statistically significant difference between both sexes, we could not prove that organochlorine concentrations in human serum are sex-dependent.

Previous reports established a relationship between age and organochlorine residues levels (Stehr-Green, 1989; Soliman et al., 1997; Voorspoels et al., 2002) that we could not statistically corroborate. However, it is important to note that younger groups have high levels of detection of analysed residues, causing suspicion of exposure to pesticide residues continues to happen.

The comparison of our results with the results obtained from monitoring studies of organochlorine residues in human serum in other countries is presented in Table 6. In relation to mean levels of $\mathrm{HCH}$ isomers and DDT isomers and analogues, higher values were achieved in Nainital, India (Dua et al., 2001), in a study performed in whole 
blood during three different months. In fact, those levels were very much higher than levels found in other countries from Asia, such as Pakistan (Krawinkel et al., 1989) and Japan (Hanaoka et al., 2002), as well as in others from Europe or America. About DDT related compounds, our study revealed higher levels of contamination than other studies performed in countries from Europe, such as Spain, Italy, Germany or Belgium. Mexico showed mean $p, p^{\prime}$ DDE levels, $14.5 \mu \mathrm{g} / 1$, comprehended among ours, lower than those found in Coimbra, $28.6 \mu \mathrm{g} / \mathrm{l}$, but higher than those found in Verride, $9.5 \mu \mathrm{g} / 1$. However, Sweden revealed mean $p, p^{\prime}$ DDE levels much higher than ours, 808.9 $\mathrm{ng} / \mathrm{g}$. Mean $\alpha-\mathrm{HCH}$ levels were highly detected in our study, while mean $\beta$ and $\lambda-\mathrm{HCH}$ levels were higher in Germany (DeVoto et al., 1998). $\beta$ $\mathrm{HCH}$ higher levels were detected in Sweden (Wicklund Glynn et al., 2000), 48.6 ng/g lipid. Also mean $\lambda-\mathrm{HCH}$ levels detected in Sweden were higher than those detected in our study. This comparison between Europe, Asia and America revealed that Asia and Europe continue to show high levels of contamination, as well as countries from South-America, while the USA and Canada showed low levels.

Although the majority of epidemiological studies, have not confirmed these chemical compounds as likely causes of diseases like cancer, the fact that almost all samples tested to date have shown detectable levels of residues of pesticides (especially $p, p^{\prime} \mathrm{DDE}$ ) provides ample reason for concern about many possible health effects of these compounds. Hence, further monitoring studies are needed in an attempt to eliminate or reduce sources of contamination, and also providing information for epidemiological studies that establish a relation between levels of contamination and predominance of certain diseases like cancer.

\section{Acknowledgments}

Besides the financial support of FCT, the authors thank our colleague Ph.D. Fernando Ramos for his precious help in samples process acquisition. $\mathrm{Dr}^{\mathrm{a}}$ Fátima Rodrigues, Health Centre Director of Montemor-o-Velho, Dr ${ }^{\mathrm{a}}$ Fernanda Palma and Dr Arriaga for giving permission for sample collection, and nurse $\mathrm{M}^{\mathrm{a}}$ Luz Batista, for collecting the samples. We also would like to thank $\operatorname{Dr}^{\mathrm{a}}$ Lucília Silveira, Isabel Alves and $\mathrm{M}^{\mathrm{a}}$ Isabel Mamede from the Laboratory of Clinical Analysis for their cooperation.

\section{References}

Ahlborg UG, Lipworth L, Titus-Ernstoff L, Hsieh CC, Hanberg A, Baron J, Trichopoulos D, Adami HO. Organochlorine compounds in relation to breast cancer, endometrial cancer, and endometriosis: an assessment for the biological and epidemiological evidence. Crit Rev Toxicol 1995; 25(6):463-531

Amaral Mendes JJ. The endocrine disrupters: a major medical challenge. Food Chem Toxicol 2002;40:781-788.

Ando M, Hirano S, Itoh Y. Transfer of hexachlorobenzene (HCB) from mother to new-born baby through placenta and milk. Arch Toxicol 1985;56:195-200.

Aprea C, Colosio C, Mammone T, Minoia C, Maroni M. Biological monitoring of pesticide exposure: a review of analytical methods. J Chromatogr B 2002;769:191-219.

Charlier CJ, Plomteux GJ. Determination of organochlorine pesticide residues in the blood of healthy individuals. Clin Chem Lab Med 2002;40(4):361 -364.

Covaci A, Manirakiza P, Schepens P. Persistent organochlorine pollutants in soils from Belgium, Italy, Greece, and Romania. Bull Environ Contam Toxicol 2002;68:97-103.

Decree 660/88, Diário da República Portuguesa, I Série No. 227, 4004, 30/9/1988.

DeVoto E, Kohlmeier L, Heeschen W. Some dietary predictors of plasma organochlorine concentrations in an elderly German population. Arch Environ Health 1998;53(2):147155.

Dua VK, Kumari R, Sharma VP, Subbarao SK. Organochlorine residues in human blood from Nainital (U.P.), India. Bull Environ Contam Toxicol 2001;67:42-45.

Frías MM, Frenich AG, Vidal JLM, Sánchez MM, Olea F, Olea N. Analyses of lindane, vinclozolin, aldrin, $p, p^{\prime}$-DDE, $o, p^{\prime}$-DDT and $p, p^{\prime}$-DDT in human serum using gas chromatography with electron capture detection and tandem mass spectrometry. J Chromatogr B 2001;760:1-15.

Gammon MD, Wolff MS, Neugut AI, Eng SM, Teitelbaum SL, Britton JA, Terry MB, Levin B, Stellman SD, Kabat GC, Hatch M, Senie R, Berkowitz G, Bradlow HL, Garbowski G, Maffeo C, Montalvan P, Kemeny M, Citron M, Schnabel F, Schuss A, Hajdu S, Vinceguerra V, Niguidula N, Ireland K, Santella RM. Environmental toxins and breast cancer on Long Island. II. Organochlorine compound levels in blood. Cancer Epidemiol Biomark Prev 2002;11:686697.

Gómez-Catalán J, To-Figueras J, Planas J, Rodamilans M, Corbella J. Pentachlorophenol and hexachlorobenzene in serum and urine of the population of Barcelona. Human Toxicol 1987;6:397-400. 
González RH. Pesticide residues in developing countries, a review of residues detected in food exports from the developing world. Ninth International Congress on Pesticide Chemistry 'The Food-Environment Challeng', 2-7 August 1998, London S 7.5.

Guardino X, Serra C, Obiolis J, Rosell MG, Berenguer MJ, López F, Brosa J. Determination of DDT and related compounds in blood samples from agricultural workers. J Chromatogr A 1996;719:141-147.

Hanaoka T, Takahashi Y, Kobayashi M, Sasaki S, Usuda M, Okubo S, Hayashi M, Tsugane S. Residuals of beta-hexachlorocyclohexane, dichlorodiphenyltrichloroethane, and hexachlorobenzene in serum, and relations with consumption of dietary components in rural residents in Japan. Sci Total Environ 2002;286:119-127.

Harris CA, Woolridge MW, Hay AWM. Factors affecting the transfer of organochlorine pesticide residues to breastmilk. Chemosphere 2001;43:243-256.

Iscan M, Coban T, Cok I, Bulbul D, Eke BC, Burgaz S. The organochlorine pesticide residues and antioxidant enzyme activities in human breast tumors: is there any association? Breast Cancer Res Treatment 2002;72:173-182.

IARC Monographs on the evaluation of carcinogenic risks to humans. Occupational exposures in insecticide application, and some pesticides, DDT and associated compounds, 53. International Agency for Research on Cancer, 1991; 179.

Koppen G, Covaci A, Cleuvenberg RV, Schepens P, Winneke G, Nelen V, Larebeke N, Vlietinck R, Schoeters G. Persistent organochlorine pollutants in human serum of 50-65 years old women in the Flanders Environmental and Health Study (FLEHS). Part 1: concentrations and regional differences. Chemosphere 2002;48:811-825.

Krawinkel MB, Plehn G, Kruse H, Kasi AM. Organochlorine residues in Baluchistan/Pakistan: blood and fat concentrations in humans. Bull Environ Contam Toxicol 1989;43:821 -826.

Leoni V, Fabiani L, Marinelli G, Puccetti G, Tarsitani GF, Carolis AD, Vescia N, Morini A, Aleandri V, Pozzi V, Cappa F, Barbati D. PCB and other organochlorine compounds in blood of women with or without miscarriage: a hypothesis of correlation. Ecotox Environ Safe 1989;17:111.

Lino CM, Silveira MIN. Contribuições para a presença de resíduos de pesticidas organoclorados no homem. Revista Portuguesa de Farmácia 1996;XLVI(4):143-147.

Lino CM, Azzolini CBF, Nunes DSV, Silva JMR, Silveira MIN. Methods for the determination of organochlorine pesticide residues in human serum. J Chromatogr B 1998;716:147-152.

Manirakiza P, Akimbamijo O, Covaci A, Adediran SA, Cisse I, Fall ST, Schepens P. Persistent chlorinated pesticides in fish and cattle fat and their implications for human serum concentrations from the Sene-Gambian region. J Environ Monitor 2002;4:609-617.

Mes J. Organochlorine residues in human blood and biopsy fat and their relationship. Bull Environ Contam Toxicol 1992;48:815-820.

Safi JM. Association between chronic exposure to pesticides and recorded cases of human malignancy in Gaza Governorates (1990-1999). Sci Total Environ 2002;284:75-84.

Saxena MC, Siddiqui MKJ, Seth TD, Krishna Murti CR, Bhargava AK, Kutty D. Organochlorine pesticides in specimens from women undergoing spontaneous abortion, premature or full-term delivery. J Anal Toxicol 1981;5:6-9.

Shaw I. Pesticides in food. Ninth International Congress on Pesticide Chemistry 'The Food-Environment Challeng', 27 August 1998, London; S 8.4.

Stehr-Green PA. Demographic and seasonal influences on human serum pesticide residue levels. J Toxicol Environ Health 1989;27(4):405-421.

Stellman SD, Djordjevic MV, Muscat JE, Gong L, Bernstein D, Citron ML, White A, Kemeny M, Busch E, Nafziger AN. Relative abundance of organochlorine pesticides and polychlorinated biphenyls in adipose tissue and serum of women in Long Island, New York. Cancer Epidemiol Biomark Prev 1998;7:489-496.

Soliman AS, Smith MA, Cooper SP, Ismail K, Khaled H, Ismail S, McPherson RS, Seifeldin IA, Bondy ML. Serum organochlorine pesticide levels in patients with colorectal cancer in Egypt. Arch Environ Health 1997;52(6):409-415.

Sonnenschein C, Soto AM, Fernandez MF, Olea N, OleaSerrano MF, Ruiz-Lopez MD. Development of a marker of estrogenic exposure in human serum. Clin Chem 1995;41/ :1888-1895.

Voorspoels S, Covaci A, Maervoet J, Schepens P. Relationship between age and levels of organochlorine contaminants in human serum of a Belgian population. Bull Environ Contam Toxicol 2002;69:22-29.

Waliszewski SM, Aguirre AA, Benitez A, Infanzon RM, Infanzon R, Rivera J. Organochlorine pesticide residues in human blood serum of inhabitants of Veracruz, Mexico. Bull Environ Contam Toxicol 1999;62:397-402.

Waliszewski SM, Aguirre AA, Infanzon RM, Benitez A, Rivera J. Comparison of organochlorine pesticide levels in adipose tissue and human milk of mothers living in Veracruz, Mexico. Bull Environ Contam Toxicol 1999;62:685690.

Wicklund Glynn A, Wolk A, Aune M, Atuma S, Zettermark S, Maehle-Schmid M, Darnerud PO, Becker W, Vessby B, Adami H. Serum concentrations of organochlorines in men: a search for markers of exposure. Sci Total Environ 2000;263:197-208.

Wolff MS, Zeleniuch-Jacquotte A, Dubin N, Toniolo P. Risk of breast cancer and organochlorine exposure. Cancer Epidemiol Biomark Prev 2000;9:271-277. 